\title{
An Analysis of Pre-Service Teachers' Reflective Think- ing for Tasks on Polar Coordinates ${ }^{1}$
}

\author{
$\mathrm{OH}, \mathrm{Hye} \mathrm{Mi}^{*}$ \\ Department of Mathematics Education, Seoul National University, \\ Seoul151-748, Korea; Email: nepscent@ hanmail.net \\ PARK, Jee Hyun \\ Department of Mathematics Education, Seoul National University, \\ Seoul151-748, Korea; Email: jeannei@ chol.net \\ KWON, Oh Nam \\ Department of Mathematics Education, Seoul National University, \\ Seoul151-748, Korea; Email: onkwon@snu.ac.kr
}

(Received May 20, 2013; Revised June 15, 2013; Accepted June 28, 2013)

\begin{abstract}
It is the first time that there is a subject, advanced mathematics in the 2009 revised high school curriculum. Therefore it is posing a challenge to the teachers who are teaching it. At the advanced level, it is important for learners to reflect on their mental mathematical activities. This research analysed pre-service secondary teachers' reflective thinking in solving the tasks specific for the teaching and learning of polar coordinates. We report how and through what process mathematical tasks that can create disequilibrium for preservice secondary teachers enable reflective thinking and expand pre-service secondary teachers' thoughts and recognition of defining reflective thinking in looking back on one's problem solving and thinking processes.
\end{abstract}

Keywords: reflective thinking, polar coordinates, pre-service teachers, mathematical tasks

MESC Classification: C34, D54

MSC2010 Classification: 97C70, 97D50

1 This paper will be presented at KSME 2013 International Conference of Mathematics Education on Creativity and Giftedness at Mokwon Univ., Doan-dong, Seo-gu, Daejeon 302-729, Korea; August 9-10, 2013.

* Corresponding author 


\section{INTRODUCTION}

The Korean mathematics curriculum has had significant changes in 2009. Several elective subjects belong to the revised high school curriculum.

Advanced mathematics is an elective subject introduced to the high school curriculum for the first time. Therefore this subject will be burden to the mathematics teachers. The reason that high school students have to learn polar coordinates in advanced mathematics is that polar coordinates can apply to many disciplines like engineering, physics and information technology. Though High school students are familiar with Cartesian coordinates, they get to be in reflective thinking by learning the different coordinate systems, polar coordinates and Cartesian coordinates. And they will get to know various mathematical expressions of the location of a point. And, they will realize flexibility of using different coordinates

Learners' reflecting on their mental mathematical activities at the advanced level has importance. Dewey (1933) explained that reflection is a meaning making process that moves a learner from one experience into the next with deeper understanding of its relationships. Piaget's (1976) construct of "reflexive abstraction," for example, is a mechanism for extracting, reorganizing, and consolidating knowledge. Piaget suggested that students were conscious of mathematics structure through reflecting their own thinking by mathematical teaching. Polya (1985), Freudenthal (1978; 1991) and Skemp's (1979) mathematical education researches also emphasized the importance of the role of reflective thinking in nurturing and involving the ability of mathematical thinking. Especially, Freudenthal emphasized that much of mathematics is about reflecting on the activities of oneself and others, and reflection is necessary for mathematization at higher levels. It is a common opinion among many mathematics education researchers that it is to be aware of one's own activities or thoughts or uses them again as objects of thinking. By doing so, it is possible to have an insight of the contents and reach the advanced level. With regard to this, in this paper, reflective thinking is regarded as looking back on one's problem solving and thinking process.

Teachers need to become familiar with reflective thinking. Dewey $(1965$, p. 152) further claimed that teachers' lack of reflective thinking leads to intellectual dependency on "those persons who give them clear-cut and definite instructions as to just how to teach this or that". Dolye (1990) argued that reflective thinking is a means to help pre-service teachers begin to view the claims from research or theory as avenues for inquiry in the face of a problematic classroom situation rather than as definite answers to all education problems. There is no research that the teaching and learning of polar coordinates has been researched in pedagogical perspectives. The purpose of this research report is to 
analyze pre-service secondary teachers' reflective thinking for tasks on polar coordinates.

\section{THEORETICAL BACKGROUND}

\subsection{Advanced mathematics}

As the environment of our society changes rapidly in recent decades, Korean mathematics curricula have been revised. There has been three times official revision of mathematics curriculum in 1997, 2007, and 2009. The 2009 revised mathematics curriculum, called 'creativity and character building curriculum', was constructed to meet the national and social demand that Korea should cultivate more creative and talented people for the globalization era. The 2009 revised mathematics curriculum focuses on mathematical creativity designed to equip students with capacities on essential learning ability, divergent thinking ability, problem-solving ability, originality, and ability to create new values. It also reinforces mathematical process including problem-solving ability, reasoning ability, and communication ability (Ministry of Education, Science and Technology, 2011).

A significant change in the 2009 revised mathematics curriculum is students' selection to mathematics subjects. It is that the high school curriculum comprises electives and allows students to choose subjects which suit their aptitude, future career and goals. The mathematics curriculum consists of three parts, that is, basic subjects (Basic Mathematics), core subjects (Mathematics I, Mathematics II, Calculus I, Calculus II, Geometry \& Vectors, and Probability \& Statistics), and advanced subjects (Advanced Mathematics I, Advanced Mathematics II), thus allowing students to choose subjects according to their levels and complete the curriculum. Students who are good at and interested in mathematics can take and complete 'Advanced mathematics I', 'Advanced mathematics II'. This allows them to give a chance to study mathematics more deeply.

Advanced mathematics is a route of advanced placement course which is an advanced course of the existing educational program and deals with new contents. As advanced mathematics has been introduced for the first time in high school curriculum, teachers who will teach advanced mathematics, need to become familiar with their materials and mathematical task that support the latest emphasis of the curriculum. In this research, feasible mathematical tasks that may help pre-service teacher nurture reflective thinking in their classrooms are introduced and analyzed.

\subsection{Polar Coordinates}

Polar coordinates have been used for special purposes and for the study of particular curves before they were unaware of a general geometrical tool. The first mathematician to 
make use of them was Bonaventura Cavalieri, who used them to find the area within an Archimedian spiral by relating it to that outside a parabola. After that, the first mathematician who considered on polar coordinates as a means of fixing any point in the plane was Newton (Coolidge, 1952). The most essential feature of polar coordinates is that the position of a point is perfectly established by the values of two parameters, a distance $(r)$ and an angle $(\theta)$.

Polar coordinate system is different from the Cartesian coordinates system, in that a point can be represented with various notations in the plane. So, the problems of a polar coordinate -e.g. to find the points of intersection - needs to be considered with that of Cartesian coordinates differently. Reflecting tasks on these types of polar coordinates problems, students get to know that they are accustomed to only the problem-solving method of Cartesian coordinates. Symmetry on polar coordinates also needs to be considered with symmetry on Cartesian coordinates differently. In Cartesian coordinates, symmetry of graphs is used in connection with algebraic equation. The method to check symmetry in polar equation is represented algebraically (Kwon, Kim, Shin \& Kang, 2011 p.121) as follows:

Consider a polar equation $r=f(\theta)$. Then

If $f(\theta)=f(-\theta)$ or $f(\theta)=-f(\pi-\theta)$, the curve is symmetrical about the polar axis ( $x$-axis).

If $f(\theta)=f(\pi+\theta)$, the curve is symmetrical about the pole.

If $f(\theta)=-f(-\theta)$ or $f(\theta)=f(\pi-\theta)$, then the curve is symmetrical about the vertical line $\theta=\frac{\pi}{2}$ (y-axis).

For example, in the case of the cardioid $r=1+\cos \theta$, since $\cos (-\theta)=\cos \theta$, the curve is symmetrical about the polar axis ( $x$-axis).

Converting geometric problems into algebraic problems is possible by using appropriate coordinates. As a result, the concept of calculus was occurred and the discipline of mathematics grew explosively. However, many students do not realize the importance of coordinates. Through experiencing polar coordinates instead of Cartersian coordinates, which students are familiar with, students will be able to involve in reflective thinking about the necessity and importance of different coordinate systems. Also, students will realize that there are various ways of showing the location of a point, thereby improving flexibility of thinking.

\subsection{Reflective Thinking}

The importance of reflective thinking in mathematics education was emphasized by John Dewey's research. Dewey (1933) described that reflection is a meaning making pro- 
cess that moves a learner from one experience into the next with more advanced understanding of its relationships. Piaget's (1976) construct of "reflexive abstraction," for example, is a mechanism for extracting, reorganizing, and consolidating knowledge. Polya (1973) suggested the importance of reflection in mathematics problem solving. The final stage of problem-solving process-looking back at the completed solution-requires students to be reflective in determining which part of their solution worked, which part did not, why it did not work and what strategy could be used to solve other similar problems in future. Skemp (1979) distinguishes between two levels of functioning of the intuitive intelligence and the reflective intelligence. He mentioned "reflective intelligence" as the "ability to make one's own mental processes the object of conscious observation" (p. 175). Freudenthal $(1978,2002)$ also emphasized that much of mathematics is about reflecting on the activities of oneself and others, and reflection is necessary for mathematization at advanced levels._Dubinsky (1991) proposed that reflective abstraction could be an important tool in the study of advanced mathematical thinking. He mentioned that reflective abstraction could provide a theoretical essence that supported and contributed to the understanding of how students think and could suggest explanations of the difficulty experienced by students with mathematical concepts. The theory on reflective abstraction by Piaget and Garcia (1989) was important for higher mathematics, as it was useful to explain children's logical thinking. In extension of this theory, Dubinsky (1991) isolated some essential features of reflective abstractions, reorganized and reconstructed them, and formed a coherent theory of mathematical knowledge and its construction, APOS. In this research, reflective thinking is considered as looking back on students' problem solving and thinking process.

Reflective thinking occurs as a cognitive action for the understanding of disequilibrium. The equilibration (Piaget, 1976, 1977) means that the results of the organism's actions look back to its psychological structure in a way that is discrepant with its structure. This discrepancy induces a state of disequilibrium, leading to reorganization of the existing structure in a way that serves to reinstate equilibrium. The mechanisms of equilibration in the learners are triggered by the disturbance of a knowledge system when a 'gap' or conflict is recognized by matching anticipation, generated by the application of it. It is not guaranteed that learners will have reflective thinking voluntarily in mathematical learning. Ge \& Land (2003) found that learners did not voluntarily construct arguments to justify their proposed solutions without being prompted to do so. Therefore, the teacher may make proper interventions to help learners do reflective thinking consciously.

The teacher may use questions intentionally to make cognitive disequilibrium for students in order to facilitate reflective thinking (Davis \& Linn, 2000; King, 1991). Dewey (1933) suggested that what is important in the first of reflective thinking is experiencing disturbance and perplexity and trying a situation. When faced with a new situation, at first 
a person receives emotional confusion, perplexity and some strange feeling, and such an emotion often leads to reflection. In other words, the problem which causes cognitive disequilibrium is discordant with the primary thinking and, and it should be able to make learners feel perplexity, strangeness or difficulty.

Table 1. The main stages of a process of reflective thinking

\begin{tabular}{|c|c|}
\hline Stage & Description \\
\hline $\begin{array}{l}\text { Stage } 0 \\
\text { Local success } \\
\text { or failure }\end{array}$ & $\begin{array}{l}\text { Initial thoughts, primary intuitions and conceptions on a subject matter or a } \\
\text { problem; starting the work with "local" success or failure; unclear and } \\
\text { "fragmentary" mental images; making observations "at random" }\end{array}$ \\
\hline $\begin{array}{c}\text { Stage } 1 \\
\text { Reflecting }\end{array}$ & $\begin{array}{l}\text { Reflecting on the subject and trying to understand, i.e. to organize the new } \\
\text { experience into previously existing intuitive structures; classifying observa- } \\
\text { tions, analyzing wholes into parts, reflecting on them, recalling other similar } \\
\text { examples, finding counterexamples, questioning former beliefs and concep- } \\
\text { tions. }\end{array}$ \\
\hline $\begin{array}{c}\text { Stage } 2 \\
\text { Discovery } \\
\text { and partial } \\
\text { understanding }\end{array}$ & $\begin{array}{l}\text { Discovery and (partial) understanding; finding and/or justifying a rule; find- } \\
\text { ing an explanation for some error; recombining parts of a decomposed } \\
\text { whole into new whole interpretation and (partial) reorganization of the new } \\
\text { facts according to previous structures; "completion" of mental images and } \\
\text { deriving a plan of the solution or of the proof; intuitive feeling of certainty } \\
\text { for the success of the plan. }\end{array}$ \\
\hline $\begin{array}{c}\text { Stage } 3 \\
\text { Introspecting }\end{array}$ & $\begin{array}{l}\text { Introspection; trying to see "what is all about" i.e. reflecting on the process } \\
\text { of the solution, the logic of the proof and one's own mental structures and } \\
\text { processes; checking or testing one's own results (or conclusions) into other } \\
\text { problems or fields; examining analogies and setting up new questions; ana- } \\
\text { lyzing the whole situation again, but at a higher level; questioning again; } \\
\text { questioning "questioning"; epistemological dispute. }\end{array}$ \\
\hline $\begin{array}{l}\text { Stage } 4 \\
\text { Full aware- } \\
\text { ness }\end{array}$ & $\begin{array}{l}\text { Full awareness; understanding the underlying logic; illumination of the } \\
\text { whole subject; becoming aware of one's own mental structures and pro- } \\
\text { cesses; widening the old structures, transforming or fully rejecting them } \\
\text { (and constructing new ones); radical reorganization of ideas, possibly on } \\
\text { new foundations; making sound generalizations and extensions; construct- } \\
\text { ing and formulating new theories. }\end{array}$ \\
\hline
\end{tabular}

Adapted from Gagatsis and Patronis, 1990, p.33

We will report how and through what process mathematical tasks that can occur to disequilibrium for learners enable reflective thinking and expand learners' thoughts and recognition by analyzing pre-service teachers' reflective thinking. Gagatsis and Patronis (1990) investigated how geometrical models can be used by learners and teacher in a process of reflective thinking. Although their model applied a process of reflective thinking to a specific geometric task, their model itself is independent of a specific mathematical content. They described the main stages of a process of reflective thinking as Table 1. So, 
we applied this model to analyze the problem solving of participant's polar coordinates tasks in this research.

\section{RESEARCH METHOD}

\subsection{Data collection and analysis}

Twenty-eight pre-service teachers were asked to complete the questionnaire with four mathematical tasks in the pilot research. To complete the questionnaire, modifications were given to let the teachers reveal their thinking processes based on the results of the pilot research. Though pre-service teachers had the math courses related to polar coordinates, Calculus and Complex Analysis, problems of polar coordinates is very difficult to pre-service teachers. And we aim to analyze the process of pre-service teacher's reflective thinking. So, the design employed for this study was case study. In case studies, researchers only observe the characteristics of individual units of research interest. The goal of observation in case study is to study the characteristics and functional pattern fo the subjects and from there make broad generalizations to the large polulation. In this study, charateristics of individual units of interest were: pre-service teacher's problem solving on polar coordinates taks. He had enough time to complete the questionnaire before the interview, and then He described his thoughts for each task for 30-40 minutes. The type of interview was the semi-structured interview, which is characterized by participant' oral explanations of his task solving process and additional questions as a means of observing their reflective thinking. For data collection, we recorded a video, conducted a voice recording, and recorded written answers, and then we analyzed one participant case by using the main stages of the process of reflective thinking as a framework.

\subsection{Interview tasks}

Kwon \& Lee (2013) proposed only the polar coordinates tasks to nurture students' reflective thinking without research design. In this research, we modified four tasks that were adapted from Kwon \& Lee's research to reveal participants' thoughts. Four interview tasks consisted of two features of polar coordinates: multi-valued expressions and symmetry. Task 1 and Task 2 asked about multi-valued expressions that a point $(r, \theta)$ in the polar coordinates could be conveyed through various expressions $\left((-1)^{n} r, \theta+n \pi\right)$ where $n$ is an integer (as in Table 2). 
Table 2. Task 1 and Task 2

\section{Task 1}

Find all points of intersection of the curves $r=\theta, r=\theta+2 \pi$

\section{Task 2}

Person A asked questions on the task-solving of all points of intersection of the curves $r=4 \cos 2 \theta, \quad r=2$. How do you answer Person A's question?

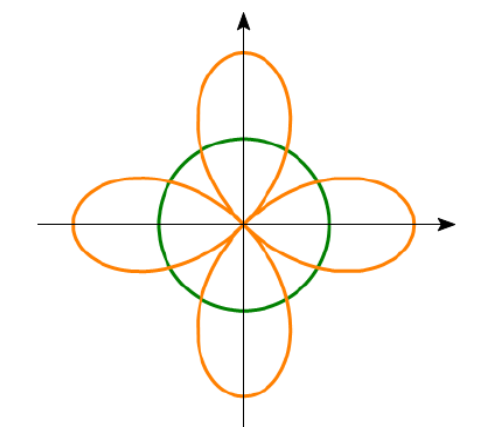

Person A's Question:

When I tried to find all points of intersection of the curves $r=4 \cos 2 \theta, r=2$, I had $4 \cos 2 \theta=2$. Since $\theta=$ $\pm \frac{\pi}{6}, \pm \frac{5 \pi}{6}$, I could find four solutions $\left(2, \pm \frac{\pi}{6}\right),\left(2, \pm \frac{5 \pi}{6}\right)$. However, this graph shows eight intersections. I did not miscalculate, so I do not know what the error is.

Task 1 and Task 2 will show the process of participants' reflective thinking that occurs while the points of intersection of the graphs are found by the system of equations in a polar coordinate. The system of equations $r=\theta$ and $r=\theta+2 \pi$ have no solution in Task 1. Students should reflect on the logic of the processes in Task 1 and analyze the whole situation again to solve Task 2 .While solving Task 1 and Task 2, students experience local success or failure when using the initial conception that intersection points on the graph of a rectangular coordinate can be found algebraically with the system of equations. Reflecting on Tasks 1 and 2, students become aware that they are accustomed to only the problem-solving method of rectangular coordinates; the problems of a polar coordinate need to be considered differently.

Task 3 and Task 4 were given to ask whether being symmetrical in the graph and checking the symmetry of polar equations algebraically is equivalent in polar coordinates as in Table 3, with regard to Task 3 and Task 4.

Reflecting on the process of drawing the graph of polar coordinates in comparison with that of Cartesian coordinates, students become aware that the difference between the two coordinates comes from the difference in the characteristics of $x$ coordinate and $\theta$ coordinate. 
Table 3. Task 3 and Task 4

\section{Task 3}

Person B asked the following:

How do you answer Person B's question? (In particular, you must point out part of Person B's misunderstanding and unknowing.)

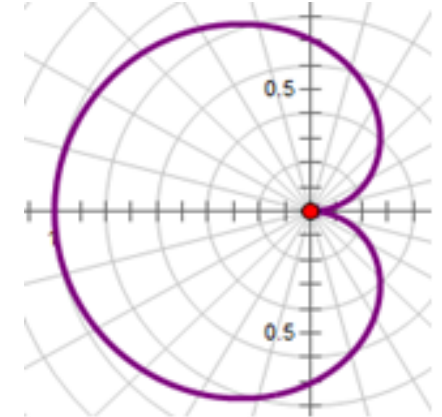

Person B's question:

The graph $f(\theta)=\sin \frac{\theta}{2}$, drawn by graphic software, shows that the graph is only symmetrical with respect to the $x$-axis. But for checking it algebraically, because $f(-\theta)=-f(\theta)$, is it symmetrical with respect to the $y$ axis?

\section{Task 4}

For this task, person C asked the following: How do you answer Person C's question?

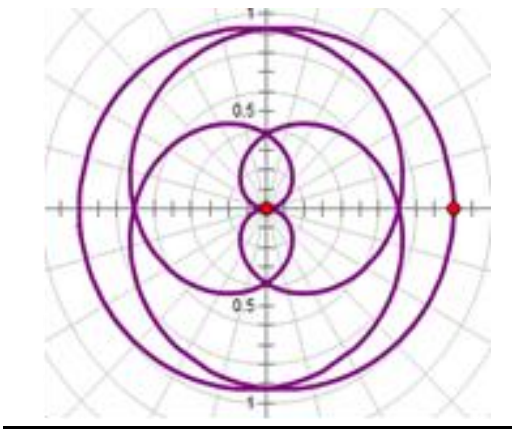

Person C's question:

I found a strange thing. The graph of $g(\theta)=\sin \frac{\theta}{4}$ is shown as being symmetrical with respect to the $x$-axis and $y$-axis. But checking algebraically, we obtained $g(\pi-\theta) \neq \sin \left(\frac{\theta}{4}\right)$ and $g(\theta) \neq g(-\theta)$. In this case, is the graph not symmetrical with respect to the $y$-axis?

\subsection{Process of reflective thinking multi-valued expressions}

Participant WJ initially tried to solve the tasks using the features of Cartesian coordinates. As he solved the tasks, he gradually reflected on his thoughts, and finally (partially) understood the features of multi-valued expressions in polar coordinates.

He solved Task 1 intuitively (Stage 0 ), and then he said that a point could be represented by $(r, \theta)$ to find the points of intersection (Stage 1). Expanding his awareness, he answered that the graph was drawn as a spiral form, and that as $\theta$ was increasing, $r$ was also increasing.

WJ: Distance between angle and origin . . . No, it seems to be enough as absolute value (Stage $0) \ldots$ I can mark some points in the thought. For $r=\theta$, if $\theta=0$, then $r=0$. So it is marked. If $\theta=2 \pi$, then $r=2 \pi$ and it is also marked equally (Stage 1). I thought I would sketch 
the graph as a spiral form like this, increasing distance to origin according to increasing angle (Stage 2).

But WJ only thought of the meaning of intersection on the graph, saying, "Since the graphs of the two equations are the same, there are multitudinous intersections." He did not think of the relationship between the solution of the equation and the intersection of the graphs.

Task 2 had the question on the solution of the equation and the intersection of graphs. WJ went further from thinking only about the intersection of the graph in Task 1, and thought of the value of $r$ without consideration, being aware that one graph could represent two polar equations (Stage 2). Therefore, he reflected on his solutions and thought of their meaning in polar coordinates, saying, "When I tried to find all points of intersection of two polar equations, I found other polar equations that represented the graph of one polar equation." (Stage 3).

WJ: It seems that the representation of one equation is not unique, unlike the Cartesian coordinate. The equations of this graph are only two: $r=2$ and $r=-2$ (Stage 2). Since uniqueness algebraically is not assured in polar coordinates, the number of solutions of the system of equations $r=2, r=-2$, and $r=4 \cos 2 \theta$ should be exactly eight (Stage 3 ).

However, WJ reflected on his mention according to $r$. He responded differently from the predicted wrong answer the graph must be drawn by an angle $\theta$ from 0 to $4 \pi$ instead of from 0 to $2 \pi$ in Task 3 (Stage 3 ). Finally, WJ failed to see his own process of solving the tasks according to $r$ and $\theta$ synthetically, thereby not reaching Stage 4 .

\subsection{Process of reflective thinking symmetry}

WJ tried to judge the symmetry of a polar equation with an algebraic equation like in the Cartesian coordinates of Task 3, even though he thought that one graph could represent one or more equations in Task 1 and Task 2. While he solved Task 4, he revised his response to Task 3. This helped him solve Task 4 . In Task 3, WJ observed ${ }^{-\theta}$ using intuition to $\theta$ (Stage 0 ) and tried to consider the meaning of $-\theta$ in regard to symmetry with respect to the axis. The meaning of $f(-\theta)=-f(\theta)$ was understood to connect with the symmetry with respect to the axis, too (Stage 1). Nevertheless, he observed the polar equation

$$
r=\sin \frac{\theta}{2}
$$

and explained errors in Task 3 according to ${ }^{\theta}$, unlike answering Task 2 according to $r$ (Stage 2). 
WJ: First, geometrically thinking, if there is $\theta$ then $-\theta$ is . . (Stage 0$),-\theta$ and $\theta$ are symmetrical with the $x$-axis. Adding $f$ to this, $f(-\theta)$ and $-f(\theta)$ should be symmetrical with the $y$ axis ... what does it happen? (Stage 1). (After thinking for a long time), since the period of $\sin \theta$ is $2 \pi$, the period of this polar equation $\sin \frac{\theta}{2}$, must be $4 \pi$. Therefore, the graph is still incomplete. If the graph is drawn right, it will be symmetrical with the y-axis (Stage 2).

WJ tried to find the symmetry of the polar equation graph in task 3 using algebraic conditions about symmetry, $f(-\theta)=-f(\theta)$ that he had used to find the symmetry of equations in the Cartesian coordinates. However, Task 4 does not satisfy algebraic conditions about symmetry, which WJ applied in Task 3. After sketching the graph of the polar equation partially and substituting some particular points for two variables $r$ and $\theta$ in the polar equation, he noticed that the polar equation did not satisfy the two algebraic conditions about symmetry. After reflecting his previous solution, he responded as follows:

WJ: Considering my solution done so far, I do not know the symmetry of the polar equation using only algebraic conditions about symmetry (Stage 3).

Subsequently, he solved Task 4 and said a feature of the polar equation is as follows:

WJ: Through the two tasks I did earlier, I am sure that the representation is not unique in polar coordinates. It seems like I learned it before. These two tasks were more difficult for me because I have no experience in judging symmetry using equations. I think that it is accurate to judge symmetry using a graph. In Cartesian coordinates, $y$ depends on $x$, but symmetry in polar coordinates is not formed sequentially since $r$ and $\theta$ are covariant, the graph of a polar equation could be drawn continuously.

As shown in the above excerpts, he figured out the features of polar coordinates (Stage 4). As the above case shows, WJ was provided the opportunity to develop his understanding of polar coordinates through tasks that cause the reflective thinking.

\section{CONCLUSIONS}

This study is intended to show that the tasks provoking cognitive disequilibrium provide students with the opportunity to complement their incomplete understanding through reflective thinking. Especially it was investigated how pre-service secondary teacher to develop their reflective thinking on a series of tasks. Summarizing mathematical tasks presented in the paper, the fundamental difference between rectangular coordinates and polar coordinates can be generalized. In rectangular coordinates, a point on the plane and an expression for it make one-to-one correspondence. As a result, generally an algebraic 
equation and the graph also make one-to-one correspondence. However, in a polar coordinate, there are numerous expressions used to refer to a point. As a result, it becomes possible to have 'many different' polar equation expressions to show a polar graph. It is clearly recognized that all other polar equations must be considered when dealing with polar graphs. Therefore mathematical tasks presented in the research are appropriate to begin for the disequilibrium and to encourage to reflective thinking.

To be able to find appropriate tasks, a teacher must have an in-depth understanding of the nature and characteristic of the mathematical concepts that s/he is supposed to teach. In addition, the role of the teacher in actual class is very important. Presenting such asks is the beginning for the disequilibrium which prompts students to have reflective thinking. In this case, the teacher must provide proper scaffolding through questioning to improve recognition of disequilibrium. Besides, s/he must be provided the opportunities to nurture their reflective thinking. This is practicable through appropriate tasks for the disequilibrium. Thereby the teacher can guide students to embody the attitude of mathematical thinking checking strategies and methods of mathematical thinking for themselves, and furthermore find the joy of mathematical discovery through experience of problemsolving independently.

\section{REFERENCES}

Boyer, C. B. (1949). Newton as an originator of polar coordinates. Amer. Math. Mon. 56(2), 7378.

Coolidge, J. L. (1952). The origin of polar coordinates. Amer. Math. Mon. 59(2), 78-85.

Davis, E. A.; \& Linn, M. (2000). Scaffolding students' knowledge integration: Prompts for reflection in KIE. International Journal of Science Education 22(8), 819-837.

Dewey, J. (1933). How we think: a restatement of the relation of reflective thinking to the educative process. Chicago: Henry Regnery Company.

Dewey, J. (1965). The relation of theory to practice in education. In: M. L, Borrowman (Ed.), Teacher education in America: A documentary history (pp. 140-171). New York: Teacher College Press. (Originally published in 1904).

Doyle, W. (1990). Themes in teacher education research. In: W. R. Houston (Ed.), Handbook of research on teacher education (pp. 3-24). New York: Macmillan. ERIC ED318735

Dubinsky, E. (1991). Reflective abstraction in advanced mathematical thinking. In: D. O. Tall (Ed.), Advanced mathematical thinking (pp. 95-123). Dordrecht, Netherlands: Kluwer. ME 1993b.02231

Freudenthal, H. (1978). Weeding and sowing: Preface to a science of mathematical education. Dordrecht, Netherlands: D. Reidel Publishing Company. ME 1979b.00145 
Freudenthal, H. (1991). Revisiting mathematics education. China lectures. Dordrecht, Netherlands: Kluwer Academic Publishers. ME 19911.01100

Gagatsis, A. \& Patronis, T. (1990). Using geometrical models in a process of reflective thinking in learning and teaching mathematics. Educ. Stud. Math. 21(1), 29-54. ME 1990h.00344

Ge, X. \& Land, S. M. (2003). Scaffolding students' problem-solving processes in an ill-structured task using question prompts and peer interactions. Educational Technology Research and Development 51(1), 21-38. ERIC EJ666611

Kwon, O. N. Kim, S. R., Shin, D. Y., \& Kang, H. J. (2011). Fundamentals of advanced mathematics (in Korean). Seoul: Seoul Textbook.

Kwon, O. N. \& Lee, J. E. (2013). Mathematical Tasks in the Advanced Mathematics Class for Nurturing Reflection. In: Beinderjeet Kaur \& Toh Tin Lam (Eds.), Nurturing Reflective Learners, Yearbook 2013, Association of Mathematics Educators. Hackensack, NJ: World Scientific. ME 2013c.00190

King, A. (1991). Effects of training in strategic questioning on children's problem-solving performance. Journal of Educational Psychology 83(3), 307-317. ERIC EJ436890

Piaget, J. (1976). The grasp of consciousness. Cambridge, MA: Harvard University Press.

Piaget, J. (1977). The development of thought: Equilibration of cognitive structures. New York: Viking.

Piaget, J. \& Garcia, R. (1989). Psychogenesis and the history of science (H. Feider, Trans.). New York: Columbia University Press. (Original work published in 1983).

Polya, G. (1985). How to Solve It: A New Aspect of Mathematical Method. Princeton, NJ: Princeton University Press. ME 1990j.01910

Skemp, R. (1979). Intelligence, learning, and action. New York: Wiley. 